\title{
Investigación-acción-jurídica: escenarios para una investigación activa y crítica en el Derecho ${ }^{1}$ \\ Investigation-legal action: scenarios for an active and critical investigation in the Law
}

\author{
DOI: http://dx.doi.org/10.17981/juridcuc.14.1.2018.10
}

Artículo de investigación. Fecha de recepción: 08/08/2018 Fecha de aceptación: 22/08/2018

\author{
Abraham Zamir Bechara Llanos \\ Corporación Universitaria del Caribe (Colombia) \\ abrahambechara@hotmail.com
}

Para citar este artículo:

Bechara, A. (2018). Investigación-acción-jurídica: escenarios para una investigación activa y crítica en el Derecho. JURÍDICAS CUC, vol. 14, no. 1, pp. 211-232. DOI: http://dx.doi.org/10.17981/ juridcuc.14.1.2018.10

\section{Resumen}

Este trabajo tiene la finalidad principal de presentar al lector los rasgos comunes que identifican el concepto de investigaciónacción, no solo para establecer los elementos básicos que en conjunto describan este modelo de investigación, sino para plantear una mirada aproximativa que permita establecer una crítica desde la concepción pedagógica en el establecimiento de herramientas teórico-prácticas que posibiliten la construcción de un escenario aplicado a la disciplina jurídica. Para esto, el enfoque de estudio, además de ser reflexivo, propenderá por establecer una perspectiva de la confrontación dialéctica entre realidad y comunidades en el establecimiento de la respuesta problémica en la experiencia práctica del investigador. Se desarrollan dos temas fundamentales: (i) la investigación acción: elementos comunes para una discusión crítica; y (ii) la investigaciónacción aplicada a la investigación jurídica. Palabras clave: investigación acción, investigación jurídica, investigación activa y crítica, realidad y trasformación

\begin{abstract}
This work aims at presenting the reader the common features that outline the concept of action research, not only to establish the basic elements that describe this research model, but also to establish a rough look that allows a critical review from the pedagogical stance in order to establish theoretical and practical tools that enable the construction of a scenario for their legal application. The approach for this study, besides being reflective, will tend to establish a dialectic confrontation between reality and communities so as to achieve a response in the practical experience of the researcher. To do so, two main topics will be expounded: (i) action research: common elements for a critical discussion; and (ii) action-research applied to legal research.

Keywords: action research, legal research, action and critical research, reality and transformation
\end{abstract}

\footnotetext{
${ }^{1}$ El presente artículo es resultado del proyecto titulado: Formación jurídica en la ciencia del derecho contemporáneo, el cual está adscrito al grupo de investigación sociojurídica GISCER, perteneciente a la línea: Derecho y formación de abogados, y vinculado a la Facultad de Derecho y Ciencias Políticas de la Corporación Universitaria del Caribe (CECAR).
}

The author; licensee Universidad de la Costa - CUC. 


\section{INTRODUCCIÓN}

La investigación-acción se presenta como un modelo pedagógico y sistemático de ver los procesos investigativos. Busca que la práctica y la experiencia docente que se desarrolla exclusivamente en escenarios y contextos educativos trascienda a la interacción con las comunidades y grupos que conforman el imaginario colectivo. Es la aproximación social de las realidades desde una vista metodológica, clara, incluyente, reflexiva y crítica, dándole una ruptura epistemológica al conocimiento buscado y enfrentado, ya no solo en el campo teórico o conceptual, sino más bien reflejado en las realidades de un mundo siempre cambiante y extremadamente complejo.

Estructurándose así, la investigación-acción, como una herramienta que propone nuevos modelos de apropiación social del conocimiento, proporciona oportunidades de cambio del establecimiento de nuevos paradigmas para la ciencia, en general, antes impensados por el establecimiento de protocolos y metodologías estrictas que solo le hacían seguimiento al plano concreto y riguroso de lo teórico. Al respecto, Ramírez (2001) presenta una clara definición del concepto de investigación-acción:

Este tipo de investigación intenta resolver preguntas que se derivan del análisis de la realidad de las comunidades y de la experiencia práctica del investigador. Los propósitos de estas investigaciones se miden por las intenciones políticas y sociales del investigador; es un sistema metodológico que pude aplicarse en la educación, para solucionar problemas en las diferentes áreas del desarrollo. Resume los diversos procesos utilizados en la metodología científica y, además de unificar criterios teóricos, define el procedimiento y los instrumentos más adecuados para realizar investigaciones productivas en poblaciones necesitadas de soluciones concretas y efectivas (pp. 43-44). 
En este orden de ideas, la investigación-acción ${ }^{2}$ propone la respuesta a los problemas tanto del saber científico, como del saber social, más en los contextos construidos desde las prácticas ${ }^{3}$ comunitarias con preeminencia a un enfoque pedagógico en la construcción de resultados que arroje la fuente de nuevo conocimiento, estableciendo así, su paralelo en la observación experimental de las causas que originan, no exclusivamente, dicotomías conceptuales sino verdaderas prácticas de unificación en el proceso de enseñanza y aprendizaje. "La investigación asume en la actualidad una función de transformación social, donde se reconoce el saber popular y la capacidad de afrontar sus propias problemáticas, lo cual equivale el compromiso, de reducir desigualdades, así como a incrementar la justicia social" (Bautista, 2011, p. 93).

En dicho contexto, este tipo de investigación se enmarca dentro de la investigación social, aquella que quiere responder a las preguntas de investigación desde una inmersión en la vida de aquellas comunidades, grupos, sectores o población que hacen parte integrante del objeto general de estudio del proceso investigativo.

\footnotetext{
${ }^{2}$ Sobre una postura crítica de los desarrollos de la investigación-acción como ruptura a los métodos tradicionales esta Alcocer (1998) al indicar la autora citada: "la investigación acción surge también como respuesta al vacío existente entre la actividad académica del investigador, los intereses políticos o económicos de la institución o empresa que contrata a este, y la población, en especial los pobres, los marginados, las mayorías. ¿Qué puede hacer el investigador que se siente comprometido con esas mayorías marginadas de ese desarrollo y pobres?, ¿Qué objeto puede tener un estudio sino lleva a transformar la realidad social para hacerla más justa, más equilibrada, mejor? Las estrategias clásicas de investigación y promoción para el desarrollo habían fracasado" (p.435).

${ }^{3}$ Para una mayor comprensión del significado de "práctica", tener en cuenta la propuesta de Paredes (2007): "el significado de una práctica está estrechamente ligado al contexto o sistema al cual pertenece, por lo tanto, no podemos comprenderla si no la relacionamos con las demás partes y con el todo" (p. 26).
} 


\section{Metodología}

El presente artículo corresponde a una investigación de corte cualitativo dentro del área del conocimiento de las ciencias sociales. El tipo de investigación está dentro del campo de la ciencia jurídica como investigación bibliográfica, desde la concepción analítica, empírica y crítica del uso de la investigaciónacción aplicada a la investigación jurídica como escenario para la construcción de una investigación activa y crítica en el derecho. Presenta una reconceptualización del paradigma clásico de investigación jurídica desde una concepción eminentemente normativista como modelo reorientado hacia la metodología para el derecho socio-jurídico, y así lograr el establecimiento de la postura epistemológica orientada al desarrollo de los problemas jurídicos desde una vista en la sociedad.

\section{La investigación-acción:}

elementos comunes para una discusión crítica

La investigación-acción, entendida también en el mundo académico como investigación-acción-participativa, conjuga tres elementos determinantes comunes para una discusión crítica. La investigación se presenta como un esquema metodológico con unos métodos, modelos, enfoques y objetos destinados a la consecución organizada y sistemática de unos fines propuestos por el interés de darle respuesta a los problemas con una mirada científica. La acción, que se genera desde la puesta en marcha de la experiencia docente, pasando por la experticia investigativa aplicando los saberes aprehendidos desde el desarrollo académico-investigativo, pero operativizandolo con la actividad del investigador en el medio en que investiga, busca más desmitificar la investigación solo vista desde el escenario común académico que interactúa directamente con el objeto de estudio propuesto en la investigación. "Esto significa que cualquier fenómeno perteneciente al ámbito de las ciencias sociales puede ser descrito como un sistema de acciones humanas que siempre puede dividirse en 'actos unidades' últimos, 
cualquiera sea el nivel de análisis empleado" (López, 2008, p. 74). Y finalmente el elemento participativo, permite que la construcción social ${ }^{4}$ del conocimiento por parte de la generación de la dualidad investigador-investigado establezca una apertura de múltiples soluciones a sus problemas, accediendo así al empoderamiento de las comunidades en sus costumbres, prácticas y saberes ancestrales. "En la investigación-acción, el investigador debe estar preparado para ser un colaborador, un socio del grupo. La relación investigador-investigados como una relación dual, deja de tener sentido, pues los grupos estudiados juegan un papel activo en el proceso de investigación" (Murcia, 2001, p. 24).

De esta manera, la investigación-acción es una fuerte herramienta de generación no solo de nuevo conocimiento, sino para la edificación de un especial instrumento de trasformación de la realidad. "La IAP constituye una opción metodológica de mucha riqueza, ya que, permite la expansión del conocimiento, genera respuestas concretas a problemáticas que se plantean los investigadores cuando deciden abordar una interrogante, y desean aportar alguna alternativa de transformación" (Colmenares, 2012, p. 104). Hoy la investigación tiene un fuerte componente social, y el enfoque principal de los métodos y modelos de investigación se orientan a las realidades sociales, pues, si bien la investigación debe responder a la conformación y consolidación de un método científico para que los resultados que de allí se generen no sean meras especulaciones o elucubraciones subjetivas, lo determinante ahora está en cómo ese conocimiento permite cambiar las realidades y la transformación de necesidades en actividades de em-

\footnotetext{
${ }^{4}$ La verdadera apuesta de la investigación-acción es su mirada social para dar respuesta a los problemas, estableciendo las técnicas y metodologías necesarias para la consecución de los fines en el desarrollo de las comunidades. Sobre esta especial relación social de la investigación acción, Yopo (1984) desarrolla este aspecto en los siguientes términos: Una investigación social para ser válida requiere de una acción conjunta de los hombres en la misma actividad que califica su esencia de hombres, es decir, "el trabajo. Es lo que se califica bajo la denominación investigación en la acción, la cual al aplicarse al proceso educativo no rechaza 'a priori' ninguna de las técnicas de investigación que usa el modelo tradicional, sino que las reubica dentro de una concepción del conocimiento dialectico que integra continuamente acción y reflexión, teoría y práctica social. De aquí que se hace necesario no olvidar que las técnicas son instrumentos para el conocimiento y la trasformación, pero jamás fines en sí mismos" (p. 42).
} 
poderamiento, desarrollo y crecimiento comunitario. "Estos procesos de investigación acción participativa surgen con la intención de acortar las grandes distancias que existen entre los avances teóricos en el campo educativo y lo que, generalmente, ocurre en la práctica cotidiana de las aulas y centros" (Ortiz y Borjas, 2008, p. 620).

He aquí el verdadero reto de la investigación en las ciencias sociales y, por ende, en la ciencia jurídica ${ }^{5}$ contemporánea. Ya aquellas investigaciones dogmáticas que no lleven un componente aplicado a la actividad social simplemente constituirían convidados de piedra frente a los problemas y dificultades de la sociedad:

Demuestra la importancia decisiva de la participación de grupos e individuos para la rentabilidad de cambios sociales, la investigación acción participativa de la que hablamos toma de la investigación participativa su insistencia en la importancia de la historia; en cambio, no busca legitimar el saber popular frente al saber dominante; promueve que las poblaciones rescaten su saber y lo desarrollen y/o transformen, en la línea de un desarrollo sostenido y armónico. De la investigación acción toma la importancia de fomentar acciones concretas de crecimiento cuanti-cualitativo, para apoyar ese desarrollo (López, 1998, pp. 15-16).

En este espacio de crítica, la investigación-acción quiere proponer más que elementos comunes para la generación de "acuerdos" del tipo conceptual o teórico que conlleven a una idea unitaria del objeto de estudio al cual se le aplica un método

\footnotetext{
${ }^{5}$ Este modelo de investigación social, concretamente el que desarrolla la investigación-acción aplicada al mundo jurídico, propone más el acercamiento del método sociológico propendiendo por la construcción del derecho a partir de la realidad social. Desde este punto es vital la posición que reflejan (Giraldo y Giraldo 2007): "en la concepción sociológica del derecho, un orden jurídico está conformado por dos clases de normas: las que definen los fines políticos del Estado, y las que establecen los medios requeridos para alcanzarlos. Ambas deben construirse a partir de la realidad social. (...) definida y delimitada la meta dentro de la realidad social, es necesario establecer las modificaciones que se deben introducir a dicha realidad para que ella se pueda alcanzar. Su objetivo final es el de formular comportamientos cuya realización conducirá al logro de la meta propuesta. (...) lo que dio lugar a la formación de escuelas que trataron de establecer nuevas técnicas en la creación, formulación e interpretación del derecho" (pp. 151-152).
} 
científico, busca la reivindicación de los métodos empíricos de aproximación y de apropiación del conocimiento mediante la superación de la figura del observador-observado, propuesta desde la filosofía clásica, para lograr una representación del observador-participante desde la perspectiva de la filosofía de la acción, donde el espectador interactúa directamente con el sujeto de estudio buscando la generación, en términos de Luhmann (2012), de una expectativa cognitiva que refleje la consecuencia de su actividad. "la distinción entre «observación» y "observación participante» es clara: la primera indica la técnica para la recopilación de datos sociales de la observación en una inmersión integral en el segmento de la sociedad estudiado" (Corbetta, 2007, p. 304).

El aporte de la investigación-acción va hacer el del establecimiento de una pedagogía social constructivista, desde el punto de vista del rediseño epistemológico del objeto de estudio, en un proceso investigativo aplicado directamente al campo de las ciencias sociales, identificando principalmente los resultados que puedan apoyar la trasformación social y el cambio de la realidad de las comunidades o grupos donde se apliquen las técnicas y procesos investigativos. "Otra de las funciones específicas de la investigación social consiste en la calcificación de los objetos que estudia (grupos, estructuras, procesos, situaciones), es decir, la ubicación de ellos en un sistema de categorías" (Briones, 1988, p. 107).

Los procesos investigativos que se desarrollan a través de la aplicación del modelo de la investigación-acción buscan, esencialmente, que la generación del conocimiento no sea propuesto por un sujeto asilado del "escenario colectivo", y que dicha validación social del conocimiento permita que no solo el investigador sea el director de la investigación en su calidad de participante activo, sino que el mismo, al objetivar su práctica constructiva en la investigación como medio para la consecución de los fines propuestos, también aprenda del mismo desarrollo de las técnicas y esquemas destinados; conocer-haciendo como agente de cambio proactivo, no solo en un campo estrictamente científico en las propuestas de intervención: 
De esta manera, la búsqueda del conocimiento se entiende como un proceso colectivo, cuyos resultados y la utilización de los mismos son obtenidos por los colectivos sociales quienes determinan el proceso de conocimiento a la vez que experimentan, en el mismo, un progreso en la maduración colectiva que permite asumir el control de los problemas que los afectan (Bautista, 2011, p. 94).

Bajo esta línea argumentativa, la metodología que quiere proponer el modelo de la investigación-acción, como proceso o procedimiento de la investigación cualitativa en las ciencias sociales, es la de generar el reconocimiento del cómo, el por qué, y el para qué investigar la realidad, proponiendo, desde la sociología, la aceptación de un conocimiento más allá del científico admitido por una comunidad minoritaria pero monopolizadora de la producción y gestación del conocimiento. "El debate científico consiste precisamente en la competencia entre programas en función de su mayor o menor grado de coherencia racional, fecundidad y capacidad heurística" (Giménez, 2004, p. 280). La investigación-acción entra directamente a intervenir el concepto de ciencia ${ }^{6}$ y la aceptación o validación del conocimiento científico suponiendo metodologías de vanguardia que reconocen que existe un conocimiento que no es menos del científico, pero que se aparta desde el punto de vista metodológico de sus técnicas, reglas, postulados y estatutos tradicionales. "Puede tratarse de investigaciones sobre la vida de la gente, las experiencias vividas, los comportamientos, emociones y sentimientos, así como al funcionamiento organizacional, los movimientos sociales, los fenómenos culturales y la interacción entre las naciones" (Strauss y Corbin, 2002, p. 12).

\footnotetext{
${ }^{6}$ La consideración de aceptar un concepto de ciencia unitario es superada con el planteamiento básico de la implementación de una metodología de investigación-acción desde la filosofía de la ciencia. Gellner (1984) indica que: "Las teorías filosóficas de la ciencia, como las incorporadas en las diversas tentativas filosóficas de demarcar el hecho científico, se esfuerzan básicamente por responder a la pregunta relativa a cómo actúa la ciencia, de qué manera se logra el gran milagro del progreso y el consenso científico" (p. 608).

Es vital también, para el proceso de una ciencia moderna bajo la propuesta desarrollada por el modelo de investigación-acción, lo planteado por Kuhn (2004), según el cual: "El surgimiento de teorías nuevas se ve usualmente precedido por un periodo de profunda inseguridad profesional debido a que exige una destrucción a gran escala del paradigma, así como grandes cambios en los problemas y técnicas de la ciencia normal. El fracaso de las reglas existentes es el preludio de la búsqueda de otras nuevas" (p. 126).
} 
La construcción, desde lo social, de una metodología de la investigación-acción hacen posibles el reconocimiento de las técnicas ancestrales de aquellas comunidades inmersas en los contextos culturales. La radicalidad del cambio va a ser tal en la transformación de las realidades circundantes a estos grupos poblacionales, que realmente se gesta una cultura de la expresión misma del pueblo ${ }^{7}$, estructurando ahora, con el apoyo de esta metodología, las posibles respuestas sociales superadas en la praxis experimental de los colectivos inmersos en dichas prácticas, instaurándose así, en palabras de Fals Borda (2014), un nuevo paradigma metodológico al que llama "ciencia popular":

Por ciencia popular -o folclor, saber o sabiduría popular- se entiende el conocimiento empírico, práctico de sentido común, que ha sido posición cultural e ideológica ancestral de las gentes de las bases sociales, aquel que les ha permitido crear, trabajar e interpretar predominantemente con los recursos directos que la naturaleza ofrece al hombre. Este saber popular no está codificado a la usanza dominante, y por eso se desprecia y relega como si no tuviera el derecho de articularse y expresarse en sus propios términos (p. 91).

\section{La investigación-acción aplicada a la investigación jurídica}

Una metodología ${ }^{8}$ de la investigación-acción aplicada a la investigación jurídica, propenderá por la superación de los escenarios de discusión y de creación del derecho desde la exclusividad del campo dogmático en la concepción de los problemas propios de la

\footnotetext{
${ }^{7}$ En este sentido, la misma expresión popular propondrá una cultura expansiva del pueblo y cómo los elementos circundantes a ellos se trasforman hacia lo positivo, instituyéndose una verdadera política cultural del "folclore", teniendo en cuenta los argumentos de Valderrama (2013) para apoyar la base teórica: "la política del folclore se refiere a los procesos sociales y culturales en los cuales sujetos se implican en acciones para disputar el significado de una representación cultural en el campo de lo folclórico" (p. 26).

${ }^{8}$ Desde una crítica al estatuto epistemológico del derecho aplicado en una metodología de la investigación científica, se sienta la posición de Sánchez (2010) de los usos esquemáticos y rígidos del mismo modelo metodológico: "Como la metodología de la investigación científica se ha desarrollado más que cualquier otro tipo de metodología, en el resto de disciplinas se la toma como modelo a seguir; no es de extrañar entonces que en el campo del derecho ocurra lo mismo. Desafortunadamente, las propuestas metodológicas que existen en la actualidad, lo hacen sin tomar en cuenta los sustentos epistemológicos de la metodología, por ello sólo se siguen esquemas de tipo formulario, de ahí que se piense que la metodología consista únicamente en rellenar los espacios en blanco de aquel formulario dado por una institución o libro" (p. 297).
} 
ciencia jurídica, construyendo así un nuevo paradigma ${ }^{9}$ desde la experiencia práctica, condición especial que permite desarrollar la investigación-acción a una del tipo socio-jurídica. "La investigación sociojurídica es, por tanto, el conjunto de supuestos epistemológicos e instrumentos metodológicos que se deben utilizar para formular el Derecho, a partir de una concepción fáctica del mismo" (Giraldo, 2005, p. 1). Al permitirse el desarrollo de una investigación de impacto social, posibilitando la construcción de nuevas herramientas que superen las teóricoconceptuales en las tradiciones investigativas soportadas en estudios dogmáticos del Derecho, no se quiere decir, que estas ya no sean importantes, pues estas soportan el sustrato teórico del derecho, pero sí se indica la necesaria participación de tipos de investigación jurídica apoyadas más en el campo social y en la validación y construcción del conocimiento jurídico en el mismo entorno social ${ }^{10}$. Con lo anterior se quiere decir que esta no solo realice procesos descriptivos de la realidad jurídica o que genere de manera satisfactoria nuevo conocimiento desde el saber disciplinar, sino que aquella aproximación o generación de nuevo conocimiento impacte de tal manera que el contexto en el que se circunscribe la investigación enfrente nuevos modelos de respuesta a los problemas sociales o jurídicos con los cuales se enfrente de manera permanente, siendo el impacto importante en la búsqueda de los problemas acuciantes al saber jurídico, el cual se apoye en un saber jurídicometodológico, esto es, que emplee nuevas herramientas en la investigación jurídica:

\footnotetext{
${ }^{9}$ La expresión paradigma puede ser aceptada como la construcción de un saber común a una pluralidad científica o académica, en el caso del derecho, la figura del jurista pude proponer la revalidación de un paradigma jurídico desarrollado desde la razón práctica como praxis del derecho. González (2003) propone una identificación del paradigma ligado a la existencia de una comunidad determinada: "Con el paradigma se diferencia una comunidad científica de otra, ya que comparten por consenso teorías y métodos que se consideran legítimos, así como los criterios para enjuiciar la validez de las soluciones propuestas" (p. 125).

${ }^{10}$ Resulta vital para una visión más amplia de la conceptualización del entorno social del conocimiento jurídico la propuesta teórica realizada por Orozco (2015), la cual tiene perspectiva histórico-sociológica: “
} 
Las definiciones de impacto social giran en torno a la idea de cómo los resultados de las investigaciones producen un cambio en el entorno social, facilitan la apropiación social del conocimiento y dan solución a problemáticas sociales, además de involucrar a los investigadores, a la universidad, directivas, profesores, estudiantes, poblaciones objeto de investigación, la comunidad en general, el Estado, las instituciones públicas y privadas (Cardona, 2012, p. 68).

Se quiere dar y proponer un paso más allá de la investigación teórica del derecho, no negando que sea importante para establecer herramientas teórico-conceptuales que reformulen ciertos temas irrefutables de nuestra disciplina legal frente a la permeabilidad de técnicas propias del lenguaje jurídico, se plantea es que estén dirigidas a la estructura social, siendo esta la que habilite la función de acción-interacción en el proceso investigativo. "La estructura social es un concepto empleado con diferentes significados, pues es definido como equivalente a sistema social o a organización social; es una población con una organización y una tecnología, que vive y se desarrolla en un medio ambiente" (Fierro, 2008, p. 196).

Esta forma de investigación permite el establecimiento de una proyección, promoción, divulgación y socialización del conocimiento que permita resolver problemas jurídicos, pero no solo desde la tradición jurídica en sentido clásico, se busca es que todas las piezas del rompecabezas jurídico encajen desde la interacción social frente a la formulación de respuestas del mismo campo de acción donde el investigador actúa con el sujeto u objeto investigado. "Los investigadores sociales, como el resto de personas que nos hablan acerca de la sociedad, son miembros de la sociedad. Estudian a los miembros de la sociedad y presentan los resultados de su trabajo a los miembros de esa misma sociedad" (Ragin, 2007, p. 34). Involucra pensar la investigación en derecho desde el medio social y no el derecho como investigación para el control social, proponiendo, por qué no, el carácter científico del 
derecho ${ }^{11}$, ya que la investigación jurídica pensada y desarrollada en el medio social, permite el empoderamiento experimental de las comunidades sobre diversos problemas agobiantes y acuciantes para esa colectividad:

El factor experimental es el que engendra de manera inmediata el derecho, pero bajo la dirección del factor racional. Está constituido por las aspiraciones a la armonía social, las cuales tienen su origen en el medio social y en la naturaleza permanente del hombre. El medio social es un medio viviente y las necesidades que experimenta deben ser satisfechas y servidas por el derecho positivo. Las aspiraciones del medio social son las que suministran la materia o sustancia misma del derecho (Pérez, 2012, p. 96).

En este entendido, el derecho de hoy es visto como una multiplicidad de conceptos y saberes que reflexionan sobre diversas teorías que tratan de explicarse qué es el Derecho, pero a esta pregunta se podría formular una respuesta: el Derecho es una gran complejidad-sistémica ${ }^{12}$ fundamentada en elementos axiológicos como principios y valores, esto desde un plano valorativo, con contenido ético y carga moral, pero que debe responder a una realidad social:

En la medida que el investigador del derecho se plantee como un aprendiz a partir de la construcción de saber en torno a un problema no territorial que tiene frente a sí mismo, podrá atravesar con él, como un vector que traspasa diversos planos, otros campos disciplinares distintos al derecho y a la historia, y podrá superar de mejor manera la fragmentación del saber, lo que exige nuevos niveles en su capacidad creativa (Botero, 2004, p. 191).

\footnotetext{
${ }^{11}$ Es importante destacar que la transdiciplinariedad en el quehacer investigativo para el Derecho, va a poner de presente un debate ya surtido con ocasión al nacimiento del Derecho a finales del siglo XIX y comienzos del siglo XX, como lo era el carácter científico del Derecho y si este podría ser considerado como ciencia. Al respecto, es válida la posición de Vásquez (2013) sobre: "acreditar el estatuto científico del derecho es un presupuesto básico sobre el cual se sostiene como construcción humana cultural, valorativa y de naturaleza mutable, lo que por obligación invade su dinamización pedagógica como escenario primario en el que se aprehenden, problematizan, reformulan, perfeccionan y avalan los conocimientos que lo componen" (p. 33).

${ }^{12}$ Para una definición más profunda del concepto de complejidad, tener en cuenta los desarrollos de Mejía y Guzmán (2002) como función determinante del sistema: "el concepto de complejidad remite a una función determinante del sistema: la función de límite. Esta representa la posibilidad de dar orden al sistema, de organizarlo internamente y, al mismo tiempo, de optimar su funcionalidad frente al entorno" (p. 40).
} 
He aquí el verdadero tema que nos atañe, la investigación en Derecho de nuestros días debe estar dentro de la misma sociedad, esto se debe a que un Derecho que se aísle de la sociedad o que no pretenda realizar cualquier tipo de regulación en apoyo a la consideración de un concepto sociológico del derecho, no estudia la ciencia jurídica en su integralidad ${ }^{13}$, ya que al pretender establecer una noción de control no puede marginarse de la sociedad misma que lo rodea y lo circunda. Lo anterior "Resulta útil para articular una visión de la sociedad, no como una totalidad compacta y homogénea sino como un conjunto de esferas sociales altamente diferenciadas" (Jaramillo, 2011, p. 413). El orden, entonces, ya no puede ser abstracto o imaginado solo en la psiquis de los legisladores o hacedores del Derecho, viviendo y recreando para el individuo realidades a medias contadas desde escenarios poco legitimados para generar un equilibrio dentro de esta gran estructura llamada sociedad, pues, el Derecho, si se piensa en el moderno, debe construir sus normas con una fuerte validación social de las mismas:

En el Derecho esta realidad se presenta de forma más palpable, pues la formación técnica o profesional es legalista, por lo que sobresale el estudio de códigos y de leyes pertinentes; lo cual, en cierto sentido, es correcto, pues los abogados -en cualquiera de sus facetas (jueces, notarios, secretarios judiciales, etc.)están condenados a depender del texto de la legislación. Sólo unos pocos estarán en la capacidad de mirar con otros ojos el rol del abogado; son ellos quienes, mediante sus escritos, realizan un significativo aporte en el Derecho (Sánchez, 2011, p. 318).

En dicha concepción, lo importante ya no será exclusivamente el estudio de la norma frente a un derecho exclusivamente normativo, aquí si el predominio de nuestros tiempos es el de un

\footnotetext{
${ }^{13}$ Para ahondar más sobre las bases del concepto sociológico del derecho y sus implicaciones en el campo teórico, ver a Krawietz (1988).
} 
Derecho ${ }^{14}$ buscado en la sociedad y desde el mismo diálogo social buscar la construcción del individuo como un ser socialmente sujeto al que el Derecho pretende regular, debiendo ser estudiado y comprendido en su complejidad existencial, no solo desde aspectos endógenos, sino recogiendo la bases exógenas de su realidad. "El diálogo social es un fin en sí mismo, constituye además un medio para la consolidación de la democracia y la formulación, ejecución y supervisión de políticas, estrategias y programas sostenibles" (Rodríguez, 2010, p. 116). Uno de ellos es el propio ser o individuo como una intersubjetividad de elementos que construyen una realidad, realidad que debe ser vista desde sus componentes sociales ${ }^{15} \mathrm{y}$ cómo estos aportes humanos construyen espacios para imaginarios colectivos que apunten siempre a la búsqueda del deber ser en una racionalidad o racionalización de los procesos sociales. Así:

La progresiva racionalización de la sociedad depende de la institucionalización del progreso científico. En la medida en que la ciencia penetre los ámbitos institucionales de la sociedad, transformando de este modo a las instituciones mismas, empiezan a desmoronarse las viejas legitimaciones (Habermas, 1986, pp. 53-54).

\footnotetext{
${ }^{14}$ Las investigaciones propiamente dichas en Derecho, o del tipo jurídicas, bajo una metodología de la investigación-acción, buscan deslindar los campos de acción de los procesos investigativos desde las mismas ramas del Derecho, no solo en su sentido tradicional, sino más allá del

mismo Derecho. Este referente va a estar defendido por Arellano (2008): "No existe razón alguna para considerar que la investigación jurídica que se emprenda no ha de exceder los linderos de una cierta rama del Derecho, de una determinada su brama jurídica o de una sola institución jurídica. Y no únicamente dejamos asentado que puede suceder y es válido que una investigación jurídica sobre un tema determinado incursione en varias ramas del Derecho, o en varias su bramas del Derecho, o, en varias instituciones jurídicas, sino que es absolutamente indispensable que, si una figura jurídica elegida para constituir el centro de atención investigatoria requiere trasladarse a varias ramas del Derecho, o a varias sub ramas o a varias instituciones jurídicas, es inconcuso que no debe ser motivo de inhibición penetrar en ramas, sub ramas o instituciones, tantas como lo exija el pleno conocimiento del tema que es motivo de las tareas investigadoras" (p. 190).

${ }^{15}$ Existe una responsabilidad del abogado y del profesional del derecho con el entorno social, con la realidad cotidiana que circunda al Derecho, pero que también lo lleva más allá del

escenario estrictamente jurídico. Según la propuesta de Carreño (2010): "El compromiso de los profesionales del derecho con su realidad social tiene profundas raíces históricas, por la misma unión entre la teoría y la realidad, pero profundamente porque la configuración de la realidad social en nuestro medio, que se erige dentro de un modelo de Estado social de derecho que en contraste con otros principios definidos en la Constitución nacional de Colombia se puede nominar como participacionista desde el ámbito democrático, lo que caracteriza fundamentalmente un sujeto comprometido con el bien común” (pp.13-14).
} 
En este entendido como gran interrogante, Luhmann (2012) se cuestionará acerca de si el Derecho moderno responde a los problemas sociales contemporáneos. Así, la teoría, como la praxis jurídica de nuestros, días debe girar en torno a la búsqueda de las respuestas a los problemas del Derecho en la sociedad ${ }^{16}$, en otras palabras, ya las respuestas jurídicas no están en el Derecho de la ley, en el Derecho de la norma, pues el Derecho como sistema únicamente normativo no alcanza a explicar las complejidades sistémicas del Derecho moderno ${ }^{17}$, ahora lo que debe interesar a los científicos del Derecho es saber un poco más de la sociedad desde la misma investigación social ${ }^{18}$ : su estructura, sus complejidades y sus posibles escenarios de relación del Derecho como subsistema del gran sistema social y otros subsistemas o pequeños sistemas que integran la sociedad como escenario complejo, como la economía, la política, la cultura, la religión, etc. Percibiendo así la sociedad:

como un sistema social que puede mantener una constancia en las relaciones de sentido entre las acciones, entre un entorno altamente complejo y contingente. Para lograr esto, tienen que hacerse ciertas selecciones dentro del sistema y organizarlo de tal manera que comprenda la alta complejidad y sea capaz de reducirla a bases de acción decidible (Luhmann, 2012, p. 148).

\footnotetext{
${ }^{16}$ Las respuestas a los problemas jurídicos contemporáneos escapan desde una estricta lógica de lo normativo, presentando nuevas herramientas para el Derecho desde la Sociología. Así, Hernández (2000) señala que: "La sociedad implica un proceso de construcción que evidencia la existencia de entramados de significación, se anuncia la posibilidad de establecer vínculos analíticos entre la que podría ser reconocida como una sociología de lo cotidiano y una etnografía interpretativa" (p. 90).

${ }^{17}$ El Derecho en la modernidad, desde el escenario estrictamente jurídico es entendido como un Estado constitucional donde se propende por la garantía y aplicación efectiva de los derechos fundamentales. Sobre este postulado en particular, Bechara (2011) ha desarrollado su trabajo.

${ }^{18}$ Desde el abordaje de los problemas sociales, se discute la objetivación de los resultados en el quehacer del investigador; sobre este referente se estructura la propuesta de Cerrillo

(2009) acerca del papel del científico social: "cada elección de una práctica metodológica con la que construir el objeto de estudio implica, a su vez, una hipótesis de partida sobre ese mismo objeto; y por supuesto, ni unas ni otras son ajenas a sus contextos de partida (incluido, por supuesto, quién financia el estudio) y al hecho de que sus resultados tendrán efectos mayores o menores- en el universo. Ninguna de las decisiones que tomamos en el proceso de investigación es ajena a la red de relaciones en la que estamos insertos por el mero hecho de ser humanos, y por consiguiente seres sociales. A los científicos sociales les viene preocupando, principalmente, la dimensión epistemológica, gnoseológica de este problema: el estatuto de la verdad, la posibilidad del conocimiento objetivo" (p. 1-2).
} 
Ya para finalizar esta construcción argumentativa, es importante proponer un ejemplo concreto desde la experiencia de la investigación jurídica aplicada. Pensemos en una comunidad, un barrio o un sector de una ciudad indeterminada que enfrente un problema ${ }^{19}$ social, que a su vez tiene consecuencias para el Derecho, como el de la drogadicción y el de la violencia urbana, o "pandillismo". A través de las herramientas de la investigación-acción se puede establecer para el Derecho una propuesta que permita, desde la socialización y promoción de los derechos humanos y fundamentales de los ciudadanos del sector, la prevención de la violencia urbana y la inseguridad ciudadana, en general, la mitigación de esta anomia social. Entonces: (i) las herramientas concretas son: inserción del investigador-participante en el objeto del problema jurídico social; (ii) la participación-observación directa de las causas sociales, culturales, económicas, políticas y jurídicas que causan el conflicto; y (iii) la solución de las problemáticas desde las herramientas propias de la comunidad como respuesta de la acción investigador-investigado, recomendadas desde un escenario conjunto y concertado.

\section{Conclusiones}

Finalmente, luego de haber construido el anterior trabajo, se puede llegar a las siguientes conclusiones: primero, los aportes planteados por el modelo de la investigación acciónaplicada a la metodología jurídica moderna y a su teoría son transcendentales no solo para entender que el derecho debe ser encontrado en la sociedad, sino para el establecimiento de un nuevo objeto de estudio del Derecho desde una nueva epistemología social. Es precisamente mediante el reconocimiento de una ciencia jurídica apoyada en una desacralización de sus aspectos formalmente normativo-investigativos e indagando por posibles soluciones a problemas sociales con

${ }^{19}$ Desde la perspectiva de problema jurídico, es importante resaltar la postura que presenta Ibáñez (1993). 
implicaciones para el Derecho desde su mixta interacción y correlación derecho-sociedad que se puede lograr esto. En esto cobra una especial relación la dimensión de la eficacia del Derecho como dimensión que pone de presente la "validación social" de las normas jurídicas en el campo de acción donde ellas pretenden responder a las acciones humanas específicas, superando el paradigma de investigación jurídica pura o básica en los componentes de formación del campo sociojurídico.

Como segunda conclusión, resulta determinante poner en evidencia que toda convivencia humana esta directa o indirectamente modelada por el Derecho, pues el orden social que tiene exclusivamente una pretensión de permanencia y durabilidad en nuestros días a veces modernos $u$ otros posmodernos es el Derecho como pretensión de ser una ciencia exclusivamente pensada en el escenario de la investigación social. La propuesta es acompañada por su teoría del rol, o de los roles, en la interacción del investigador como observador-participante, en la cual, la investigación jurídica propondría la posición del abogado como un investigador frente a la búsqueda de las posibles causas, no solo aisladamente, de los litigios o de los hechos origen de los conflictos, sino del empoderamiento de comunidades a través de sus conocimientos ancestrales y populares. Dadas las expectativas que se logren desarrollar, así serán sus repercusiones o implicaciones para el Derecho mismo. La idea de encontrar siempre herramientas que posibiliten la construcción de un conocimiento científico en el mundo jurídico permiten identificar los rasgos comunes de una investigación crítica para el Derecho de nuestros días, permitiendo así, como componente activo, el mismo proceso que se desarrolla con la aplicación de un método científico, demostrando o contrastando teorías, validando hipótesis y justificando siempre los mejores argumentos para la comprensión de fenómenos jurídicos vistos más desde la realidad social y no desde la realidad meramente dogmática o normativa. 
Como tercera conclusión, desde el plano específico de la investigación científica, la integración del Derecho y la Sociología como reconstrucción del primero dentro de una investigación acción-aplicada este, llamaría a la reivindicación permanente de una Sociología del Derecho tomada en serio. Esto implica pensar el mismo Derecho dentro de la investigación, pues al establecer el modelo de la acción en la investigación jurídica, se formulan nuevas fuentes de estudio para este, así como la misma intensión de describir la estructura social que lo rodea, posibilitando, de esta manera, la reconceptualización de un Derecho más cercano a la realidad desmitificada de los formalismos jurídicos-investigativos tradicionales.

\section{REFERENCIAS}

Alcocer, M. (1998). Investigación acción participativa. En, L. Galindo (Coord.),Técnicas de investigación en sociedad, cultura y comunicación. 433-463. México, D.F.: Pearson.

Arellano, C. (2008). Métodos y técnicas de la investigación jurídica. Cuarta edición. México, D.F.: Porrúa.

Bautista, N. (2011). Proceso de la investigación cualitativa. Bogotá, D.C.: Manual Moderno.

Bechara, A. (2011). Estado constitucional de derecho, principios y derechos fundamentales en Robert Alexy. Saber Ciencia y Libertad, 6(2), 63-76.

Briones, G. (1988). Epistemología y metodología de la investigación social. Segunda edición. Bogotá, D.C.:

Botero, A. (2004). Nuevos paradigmas científicos y su incidencia en la investigación jurídica. Revista de Derecho, Universidad del Norte, (21), 163-199.

Cardona, E. (2012). Reflexión en torno a los indicadores de impacto social en la investigación jurídica y socio-jurídica. Ratio Juris, 7(15), 61-80.

Carreño, M. (2010). Compromiso social de los profesionales del derecho. Ambiente Jurídico, 12 [Editorial], 13-16. 
Cerrillo, J. (2009). El intermediario imposible. Algunas reflexiones en torno a epistemología y ética en la investigación cualitativa. Nómadas, (24), 1-15.

Colmenares, A. (2012). Investigación-acción participativa: una metodología integradora del conocimiento y la acción. Voces y Silencios, 3(1), 102-115.

Corbetta, P. (2007). Metodología y técnica de investigación social. Trad. Marta y Susana Díaz Ugarte. : Mac Graw Hill.Fals Borda, O. (1990). El problema de cómo investigar la realidad para transformarla por la praxis. Octava edición. Bogotá, D.C.: Tercer Mundo Editores.

Fals Borda, O. (2014). La ciencia y el pueblo: nuevas reflexione sobre la Investigación-Acción (Participativa). En, N. Herrera y L. López (Comp.), Ciencia, compromiso y cambio social. (301-320). Montevideo: Colección Pensamiento Latinoamericano. Recuperado de http://www.documentos.una. ac.cr/bitstream/handle/unadocs/8133/Ciencia\%2C\%20 compromiso $\% 20 y \% 20$ cambio $\% 20$ social_Orlando $\% 20$ Fals\%20Borda.pdf?sequence $=2 \&$ isAllowed $=\mathrm{y}$

Fierro, H. (2008). El argumento y la fundamentación jurídica. Bogotá, D.C.: Leyer.

Gellner, E. (1984). El rango científico de las ciencias sociales. Revista internacional de Ciencias Sociales, Epistemología de las Ciencias Sociales, 36(4), 601-622.

Giménez, G. (2004). Pluralidad y unidad de las ciencias sociales. Estudios sociológicos, 22(65), 267-282.

Giraldo, J. y Giraldo, O. (2007). Metodología y técnica de la investigación jurídica. Décima edición. Bogotá, D.C.: Ediciones del profesional.

Giraldo, J. (2005). Los supuestos teóricos de la investigación sociojurídica. Recuperado de https://docplayer.es/20776028Los-supuestos-teoricos-de-la-investigacion-sociojuridica. html

González, A. (2003). Los paradigmas de investigación en las ciencias sociales. Islas, (45), 125-135. 
Habermas, J. (1986). Ciencia y técnica como ideología. Manuel Jiménez Redondo (trad.). Madrid: Tecnos.

Hernández, D. (2000). Cultura y vida cotidiana. Apuntes teóricos sobre la realidad como construcción social. Revista Sociológica, 15(43), 87-102.

Ibáñez, B. (1993). El problema jurídico: importancia y ayudas para su correcto planteamiento. Revista de Derecho, Universidad del Norte, (2), 75-80.

Jaramillo, J. (2011). Bourdieu y Giddens. La superación de los dualismos y la ontología relacional de las prácticas sociales. Revista CS, (7), 409-428.

Krawietz, W. (1988). El concepto sociológico del derecho. Doxa. Cuadernos de Filosofía del Derecho, (5), 253-274.

Kuhn, T. (2004). La estructura de las revoluciones científicas. Carlos Solís Santos (trad.). Segunda edición. México, D.F.: Fondo de Cultura Económica.

López, P. (1998). Un método para la investigación-acción participativa. Madrid: Popular.

López, D. (2008). El problema de la subjetividad en la explicación sociológica. Una mirada a partir del debate Schütz-Parsons. Revisa de estudios sociales, (31), 72-83.

Luhmann, N. (2012). Sociología del Derecho. Londres: Routledge \& Kegal Paul.

Mejía, O. y Guzmán, A. (2002). La corte constitucional: entre la emancipación social y la eficacia sistémica. Revista Pensamiento Jurídico, (15), 37-50.

Murcia, J. (2001). Investigar para cambiar un enfoque sobre investigación-acción participante. Tercera edición. Bogotá, D.C.: Editorial Magisterio.

Ortiz, M. y Borjas, B. (2008). La investigación acción-participativa: el aporte de Fals Borda a la educación popular. Espacio Abierto, 17(4), 615-627.

Paredes, G. (2007). Críticas epistemológicas y metodológicas a la concepción positivista en las ciencias sociales. Revista Academia, 6(12), 24 -42. 
Pérez, J. (2012). Metodología y técnica de la investigación juridica. Bogotá, D.C.: Temis.

Ragin, C. (2007). La construcción de la investigación social. Introducción a los métodos y su diversidad. Bogotá, D.C.: Siglo del Hombre Editores.

Ramírez, E. (2001). La investigación socio-jurídica. Bogotá, D.C.: Doctrina y Ley.

Rodríguez, J. (2010). El diálogo y la concertación social. Estudios de Derecho, 67(150), 113-134.

Sánchez, M. (2010). Apuntes para una metodología jurídica: la idea de marco teórico. Revista Telemática de Filosofía del Derecho, (13), 297-310.

Sánchez, M. (2011). La metodología en la investigación jurídica: características peculiares y pautas generales para investigar en el derecho. Revista Telemática de Filosofía del Derecho, (14), 317-358.

Strauss, A. y Corbin, J. (2002). Bases de la investigación cualitativa. Medellín: Editorial Universidad de Antioquia.

Valderrama, C. (2013). Folclore, raza y racismo en la política cultural e intelectual de Delia Zapata Olivella. En el campo político-intelectual afrocolombiano. Revista CS, (12), 259-295.

Vásquez, J. (2013). De la indisciplina a la interdisciplinariedad del derecho. Temas sociojurídicos, 32(65), 13-35.

Yopo, B. (1984). Metodología de la investigación participativa. Cuadernos del CREFAL, (16), 1-63. 


\begin{abstract}
Abraham Zamir Bechara Llanos es Abogado summa cum laude y especialista en Derecho constitucional de la Universidad Libre de Colombia. Magíster en Derecho magna cum laude de la Universidad del Norte. Doctor en Derecho de la Universidad Autónoma de Barcelona, España. Profesor investigador del programa de Derecho de la Corporación Universitaria del Caribe (CECAR), Sincelejo.
\end{abstract}

